\title{
Os jovens brasileiros e a sua preferência pela disciplina Ciências
}

\author{
Young brazilians and their preference for Sciences discipline \\ Los jóvenes brasileños y su preferencia por la disciplina de las Ciencias
}

\author{
Kauana Gehrke Tonin \\ ORCID: https://orcid.org/0000-0001-9788-7231 \\ Universidade Federal de Santa Maria, Brasil \\ E-mail: kauanatonin@gmail.com \\ Luiz Caldeira Brant de Tolentino- Neto \\ ORCID: https://orcid.org/0000-0001-6170-1722 \\ Universidade Federal de Santa Maria, Brasil \\ E-mail: 1caldeira@gmail.com \\ Daniel Morin Ocampo \\ ORCID: https://orcid.org/0000-0003-1136-4654 \\ Universidade Federal de Santa Maria, Brasil \\ E-mail: kavu_br@yahoo.com.br
}

\begin{abstract}
Resumo
O projeto ROSE (The Relevance of Science Education) busca compreender dados atitudinais e emocionais de jovens acerca da Ciência e Tecnologia a partir de dados coletados em um questionário já aplicado em mais de 40 países. Os resultados do ROSE-Brasil mostraram que os jovens apresentam um alto interesse pela disciplina ciências, mas que gostam mais de outras disciplinas. Este artigo tenta compreender como os jovens brasileiros se comportam frente à essa divergência ao redor do interesse e da preferência pela disciplina de ciências. Para alcançar nosso objetivo utilizamos os dados brutos da amostra ROSE-Brasil 2011, e nos valeremos de métodos de análises estatísticas. Os jovens brasileiros dividem-se em quatro grupos: Prioridade não específica (3,98\%), esse grupo não tem interesse pelas aulas de ciências, mas prefere a disciplina a outras; Baixa prioridade (20,26\%), não possuem nem interesse, nem preferência pela disciplina ciências; Prioridade específica $(30,6 \%)$ possuem interesse e preferem a disciplina ciências a outras; Outras prioridades $(45,14 \%)$ grupo mais expressivo encontram-se os jovens que têm interesse mas não preferem a disciplina ciências. Desses grupos três são mais representativos da realidade dos jovens brasileiros, tornando relevante investigar as diferenças entre os interesses e hábitos ligados à ciência e tecnologia dos jovens de cada grupo para subsidiar a criação de estratégias que tornem o ensino de ciências mais atrativo.
\end{abstract}

Palavras-chave: ROSE Brasil; Interesse pela ciência; Avaliação em larga escala; Ensino.

\begin{abstract}
The ROSE project (The Relevance of Science Education) seeks to understand attitudinal and emotional data of young adults regarding the subjects Science and Technology collected from a questionnaire applied in over 40 countries. The results of ROSE-Brazil showed that while young adults have higher interest in the sciences, they like other disciplines better. This study aims to understand how young Brazilians behave in the face of this divergence around interest and preference for the science discipline. To achieve our goal, we used the raw data from the ROSE-Brazil 2011 sample and the statistical analysis methods. Young Brazilians are divided into four groups: Not-positive priority (3.98\%) group has no interest in science classes, but prefers this discipline to others; Poor priority (20.26\%) group has neither interest nor preference for the science discipline; Specific priority (30.6\%) group is interested and prefers the science subject to others; Other priority (45.14\%) is the most expressive group whose young adults are interested in the science discipline, but do not prefer it. Three of these groups are more representative of the reality of young Brazilians. For this reason, investigating the differences between the interests and habits of young people in each group linked to the subjects Science and Technology is relevant in order to help create strategies that make science teaching more attractive.
\end{abstract}

Keywords: ROSE Brazil; Interest in science; Large scale evaluation; Teaching.

\section{Resumen}

El proyecto ROSE (The Relevance of Science Education) búsqueda comprender datos de lás actitudes y emociones de los jóvenes sobre ciencia y tecnología a partir de datos colectados en un cuestionario ya aplicado en más de 40 países. Los resultados de ROSE-Brasil mostraron que los jóvenes tienen un alto interés por la disciplina científica, pero les gustan más otras disciplinas. Este artículo trata de comprender cómo se comportan los jóvenes brasileños ante esta divergencia al rededor del interés y preferencia por la disciplina científica. Para lograr nuestro objetivo, utilizamos los datos brutos de la muestra ROSE-Brasil 2011 y utilizamos métodos de análisis estadístico. Los jóvenes brasileños se 
dividen en cuatro grupos: Prioridad no específica (3,98\%), este grupo no tiene interés en las clases de ciencias, pero prefiere la disciplina a otras; Prioridad baja (20,26\%), no tienen interés ni preferencia por la disciplina científica; Prioridad específica $(30,6 \%)$ están interesados y prefieren la disciplina de ciencias a otras; Otras prioridades $(45,14 \%)$ el grupo más expresivo son los jóvenes que están interesados pero que no prefieren la disciplina científica. De estos grupos, tres son más representativos de la realidad de los jóvenes brasileños, por lo que es relevante investigar las diferencias entre los intereses y hábitos vinculados a la ciencia y la tecnología de los jóvenes en cada grupo para apoyar la creación de estrategias que hagan más relevante la enseñanza de la ciencia más atractiva.

Palabras clave: ROSE Brasil; Interés por la ciencia; Evaluación a gran escala; Ensenãnza.

\section{Introdução}

Em muitos países percebe-se um crescente desinteresse pela ciência escolar e pela carreira científica (Schreiner \& Sjøberg, 2004; SJøberg, 2004; Jenkins \& Nelson, 2005; Matthews, 2007; Vázques \& Manassero, 2008). A Europa, desde 2001, se dedica a tentar melhorar os índices de interesse dos estudantes nas áreas de matemática, ciências e tecnologia (MCT), por meio de implementações de estratégias para melhorar o ensino nessas áreas (Kearney, 2011). Mesmo com os esforços, em 2016 um novo relatório aponta que a maioria dos países europeus ainda enfrenta um baixo interesse dos alunos em estudar ou seguir carreiras nas áreas científicas (STEM - Ciência, Tecnologia, Engenharia e Matemática) (Kearney, 2016).

Essa falta de interesse parece ser um processo gradual ao longo da escolaridade que tem seu ponto de virada (interesse para desinteresse) na adolescência, a qual coincide com o fim dos estudos compulsórios, e parece afetar mais as meninas (Vázques \& Manassero, 2008). Acredita-se que essa falta de interesse está ligada à forma de ensino tradicional e expositiva dos conteúdos que pouco se conectam com a realidade dos estudantes (Pozo \& Crespo, 2009).

O currículo e as estratégias de ensino das aulas de ciências influenciam no interesse dos alunos pela disciplina, tendo o professor um papel central. Os alunos em contextos diferentes como Islândia e Brasil, reconhecem a importância do professor de ciência nas suas aulas (Stefánsson, 2006; INEP, 2016).

Os jovens brasileiros reconhecem os esforços dos professores de ciência em mediar a aprendizagem (INEP, 2016), assim como veem na imagem dos professores uma fonte de informação confiável com quem podem conversar sobre Ciência \& Tecnologia (C\&T) (INCT-CPCT, 2019).

Já os jovens da Islândia reconhecem que o método utilizado para mediar a aprendizagem em ciências é importante e varia conforme o professor (Stefánsson, 2006). Quando questionados o que poderia melhorar as aulas de ciências eles citam uma metodologia mais ativa com trabalhos práticos, discussões e menos currículo orientado por conteúdo (stefánsson, 2006). Entendem ainda, que métodos tradicionais são mais eficazes quando a preparação visa os exames nacionais.

Tanto o currículo quanto os métodos de ensino sofrem pressão de avaliações em larga escala, externas à escola, sejam elas nacionais ou internacionais. Os exames nacionais, podem desempenhar diferentes funções como: avaliar a qualidade do ensino, a aprendizagem (caso do SAEB) e até servir como instrumento para ingresso em níveis superiores de ensino (como o ENEM no Brasil). As avaliações internacionais são instrumentos aplicados em diferentes países, e tem como finalidade mensurar o conhecimento dos estudantes em determinadas áreas servindo também como fonte de comparação entre países.

Dentre essas avaliações internacionais em larga escala podemos citar o PISA (Programa Internacional de Avaliação do Estudante), programa da OCDE (Organização para Cooperação e Desenvolvimento Econômico). Essa avaliação tem como objetivo mensurar o nível de letramento de estudantes de 15 anos de idade nas áreas de linguagem, matemática e ciências (INEP, 2016). O Brasil participa do PISA como país convidado desde sua primeira aplicação no ano de 2000.O PISA é aplicado trienalmente sendo que em cada aplicação é dado um enfoque maior em uma das áreas - nos anos de 2006 e 2015 o enfoque recaiu sobre ciências. 
O ranqueamento do desempenho dos estudantes de cada país é o resultado mais conhecido, difundido e explorado em cada edição do PISA. Sendo a comparação entre o ranqueamento de um país com seus pares motivo para crises em sistemas educacionais ao redor do mundo.

O desempenho médio dos estudantes brasileiros em ciências, quando comparados aos dos países membros da OCDE, encontra-se abaixo da média, sendo que a grande maioria dos jovens brasileiros se encontram no primeiro nível de proficiência em ciências (INEP, 2008; INEP, 2016).

Os níveis de proficiência em ciências funcionam em uma escala de sete níveis, indo do menor nível (nível 1b) ao maior nível (nível 6) (INEP, 2016). Cada nível possui uma descrição contendo os conhecimentos e habilidades exigidas dos estudantes para alcançar aquele nível. Os alunos do nível $1 \mathrm{~b}$ atingem os requisitos desse nível, mas não conseguem atingir os requisitos do próximo nível. Enquanto os alunos do nível 6 cumprem os requisitos daquele nível e possuem uma alta probabilidade de cumprir os requisitos dos níveis abaixo dele (INEP, 2016).

Nos anos em que a ênfase recaiu em ciências, além de mensurar o conhecimento na área, também ocorreu a aplicação de questionário para compreender as atitudes dos jovens em relação à ciência. Em ambos os anos os jovens brasileiros demonstram atitudes positivas em relação à ciência, sendo a atitude dos brasileiros mais positiva que a média dos membros da OCDE (INEP, 2008; INEP, 2016).

Outra fonte que corrobora estas atitudes positivas dos jovens brasileiros em relação à ciência é o questionário ROSE Brasil aplicado em 2011 por Santos-Gouw (2013). O questionário faz parte do projeto ROSE (The Relevance of Science Education), que busca ouvir a voz dos estudantes sobre as suas atitudes e emoções acerca da Ciência e Tecnologia (C\&T), o qual conta com a participação de mais de 40 países (Schreiner \& Sjøberg 2004). Não é um teste de desempenho, mas de percepção/opinião sobre aspectos ligados à educação científica e tecnológica.

No estudo de Santos-Gouw (2013) a autora aponta que os jovens brasileiros possuem interesse em aprender sobre C\&T, e acham as suas aulas de ciências interessantes. Porém ciências não é considerada uma das suas disciplinas preferidas.

Ogawa e Shimode (2004) apresentam que os jovens japoneses possuem essa mesma divergência entre ter interesse nas aulas de ciências, mas não considerar a disciplina de ciência como sua preferida. Para os autores, ter interesse nas aulas de ciências é considerado um interesse absoluto, uma vez que a pergunta é direcionada apenas para as aulas de ciências. Já gostar mais da disciplina de ciências do que de outras disciplinas é considerado interesse relativo na disciplina, pois ocorre comparação. Para Ogawa \& Shimode (2004) a combinação dessas duas variáveis, interesse absoluto e interesse relativo, vem a formar um terceiro ângulo de análise, a preferência pela disciplina ciências.

A pesquisa de Ogawa e Shimode (2004) serviu de inspiração para o estudo aqui proposto, trazido para o contexto brasileiro do início da segunda década do século XXI.

\subsection{O Projeto ROSE}

O ROSE é um projeto internacional que surgiu com intuito de compreender como os jovens se relacionam com a Ciência \& Tecnologia e com suas aulas de ciências (Schreiner \& Sjøberg, 2004). Segundo os autores, a ideia é que essas informações possam servir de subsídio para que se discutam questões importantes que rodeiam a educação em ciências. Esse projeto conta com a colaboração de diversos pesquisadores ao redor do mundo, sendo a sua sede na Universidade de Oslo na Noruega (Santos-Gouw, 2013). Para atingir os objetivos do projeto foi elaborado o questionário homônimo ao projeto, que coletou dados de jovens que estão finalizando os estudos compulsórios (em média 15 anos) sobre a sua percepção em relação à C\&T (Schreiner \& Sjøberg, 2004). 
O questionário ROSE é flexível no sentido de que, em cada país, em cada contexto, pode ter seções acrescidas ou suprimidas. Ainda assim, de forma geral ele tem uma parte inicial que visa coletar dados básicos como idade, sexo e cidade, uma parte final destinada a questões socioeconômicas e uma parte central contendo 8 seções.

As seções que correspondem a parte central são: "O que eu quero aprender" (Seção A, C e E) visa conhecer os temas de maior e menor interesse na aprendizagem de ciências; "Meu futuro emprego" (Seção B) coleta informações sobre prioridades e motivações futuras; "Eu e os desafios ambientais"(Seção D) captar atitudes e opiniões perante ao meio ambiente e aos problemas ambientais; "Minhas aulas de Ciências" (Seção F) compreender as percepções sobre as aulas de ciências; "Minhas opiniões sobre ciências e a tecnologia" (Seção G) como o jovem percebe a relação Ciência, Tecnologia e Sociedade; "As minhas experiências fora da escola" (Seção H) obter informações sobre atividades realizadas fora da escola que possam influenciar na aprendizagem em C\&T. Essas seções possuem ao todo 245 afirmativas (Schreiner, 2006).

As seções são compostas por afirmativas onde os jovens, devem a partir de uma escala de Likert de 4 pontos, marcaram seu nível de interesse ou concordância com a alternativa. A escala de Likert de 4 pontos, ao contrário da escala de Likert de 5 pontos, visa evitar um posicionamento neutro do respondente o que pode levar a erros de interpretação (Schreiner \& Sjøberg, 2004). Nesta escala 1 é o menor escore e pode corresponder a discordo totalmente, sem interesse ou nunca, e o 4 é o maior escore correspondendo a concordo totalmente, muito interessado ou com frequência (Schreiner, 2006). A imagem (Figura 1) abaixo ilustra uma seção do ROSE Brasil com duas de suas questões.

Figura 1 - Imagem do cabeçalho da Seção F do questionário ROSE Brasil com questões desta mesma seção.

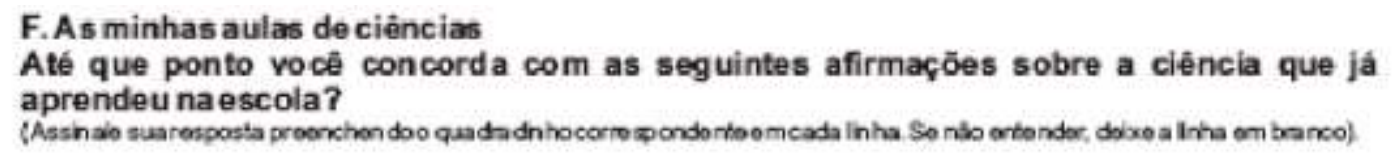

\begin{tabular}{|c|c|c|c|c|c|}
\hline & & 1 & 2 & 3 & 4 \\
\hline 1 & A disciplina Clencias aborda contedodas difíceis & $\square$ & $\square$ & $\square$ & $\square$ \\
\hline 2 & A disciplina Ciencias é in teressante & $\square$ & $\square$ & $\square$ & $\square$ \\
\hline 3 & As Clências, para nim, sâo bastanta fáceils de aprender & $\square$ & $\square$ & $\square$ & $\square$ \\
\hline 4 & As Ciências abriram-me os olhos para empregos novos e emocionantes & $\square$ & $\square$ & $\square$ & $\square$ \\
\hline 5 & Gosto mais de Ciéncias do que das outras disciplinas & $\square$ & $\square$ & $\square$ & $\square$ \\
\hline
\end{tabular}

Fonte: Autores.

\subsection{O ROSE Brasil}

O ROSE no Brasil contou com duas aplicações, a primeira em 2006 por Tolentino-Neto (2008), responsável também pela tradução e adaptação do questionário para o português e a realidade brasileira, e a segunda aplicação em 2011 por SantosGouw (2013), ambas as aplicações compõem as teses de doutoramento dos pesquisadores.

O questionário ROSE Brasil, possui, além das partes já citadas no questionário original, mais 23 questões consideradas "Questões Nacionais" (Santos-Gouw, 2013). A primeira aplicação contou com uma amostra de 652 alunos de duas cidades brasileiras, Tangará da Serra/MT e São Caetano do Sul/SP, com realidades socioeconômicas distintas (TolentinoNeto, 2008).

Já a segunda aplicação - utilizada nesta pesquisa - contém uma amostra representativa do país totalizando 2.365 alunos de todas as regiões brasileiras, os questionários foram respondidos por jovens na faixa etária de 15 anos pertencentes ao 
$1^{\circ}$ ano do Ensino Médio. Destes 42,33\% são homens e 57,67\% são mulheres. As escolas contempladas pela pesquisa são de sua maioria urbanas e pertencentes à rede Pública Estadual, porém a amostra também contempla escolas de zona rural, da rede pública federal e da rede privada (Santos-Gouw, 2013).

Segundo Santos-Gouw (2013), os jovens brasileiros em relação a suas aulas de ciências demonstram uma atitude geral positiva, que contrasta com a tendência encontrada nos jovens de países desenvolvidos, que não possuem uma atitude positiva em relação às suas aulas de ciências. Os dados encontrados demonstram, também, que os jovens brasileiros consideram a disciplina interessante, e percebem que a disciplina pode trazer algumas vantagens diretas da sua aprendizagem, porém de maneira geral não percebem a relação da disciplina com sua futura carreira.

Os resultados destacados anteriormente pertencem a seção F "As minhas aulas de ciências". Dentro dessa seção salientamos ainda o resultado de duas questões que serviram de motivação para a realização desta pesquisa: o alto interesse absoluto (questão F2) e um baixo interesse relativo pela disciplina de ciências (questão F5). A questão "A disciplina Ciências é interessante" (F2) possui média global de 3,17 sendo significativamente maior entre as meninas $(3,26)$ em comparação com os meninos $(3,06)$. Para a questão "Gosto mais de Ciências do que de outras disciplinas" a média dos jovens brasileiros é de 2,10 não havendo diferença significativa entre os sexos (Santos-Gouw, 2013).

Como podemos observar nos dados trazidos por Santos-Gouw (2013), os nossos jovens possuem um alto interesse na disciplina sendo sua média muito superior a 2,51 , já quando questionados sobre gostar mais da disciplina em relação a outras, essa média fica abaixo de 2,5 o que demonstra uma baixa preferência pela disciplina.

Esta pesquisa partiu da inquietação entre as diferenças encontradas nas respostas dos jovens brasileiros quanto às suas preferências pela disciplina de ciência: consideram a disciplina interessante, porém não é a preferida. Com isso objetivamos entender o comportamento dos jovens brasileiros em relação às suas preferências pela disciplina de ciências.

\section{Metodologia}

Essa pesquisa é de natureza básica, com o objetivo de gerar novos conhecimentos úteis para o avanço da ciência, e de abordagem descritiva. Segundo Gil (2002), a pesquisa descritiva tem finalidade de descrever características de uma população e geralmente se vale da coleta de dados por meio de instrumentos como os questionários. Quando a população-alvo da pesquisa é bastante numerosa costuma-se utilizar para coleta de dados a abordagem quantitativa. Essa abordagem não é muito valorada ou mesmo utilizada na área de educação (Gatti, 2004), porém esse tipo de abordagem costuma fornecer uma visão geral dos resultados, servindo para trabalhar com grandes universos de dados (Minayo, 2000). Ocampo \& Tolentino-Neto (2019, p. 35), trazem ainda que, a essa abordagem “[...] desempenha um papel fundamental na geração de visões gerais da realidade, muitas vezes com foco em amostras representativas de uma determinada população [...]". Sabemos que as pesquisas quantitativas possuem limitações em sua natureza de análise, no entanto acreditamos que são excelentes ferramentas na geração de pesquisas que servem de base para outras pesquisas e até mesmo para elaboração de políticas públicas.

Esse trabalho, utilizou o banco de dados coletados pelo ROSE Brasil em 2011. Os dados foram obtidos por meio de um convênio de cooperação técnica entre o EDEVO - Darwin, USP (Núcleo de Pesquisa em Educação, Divulgação e Epistemologia da Evolução "Charles Darwin da Universidade de São Paulo) e o PPGECQVS, UFSM (Programa de PósGraduação Educação em Ciências: Química da Vida e Saúde, Sede Universidade Federal de Santa Maria). A amostra aqui utilizada é uma amostra significativa da realidade brasileira, e para que possamos compreender os dados, nos valeremos do Software IBM SPSS (Statistical Package for the Social Science). Neste trabalho foram utilizados para análise os escores brutos de duas questões, ambas pertencentes à seção F “As minhas aulas de ciências” são elas: a questão F2 e a questão F5.

\footnotetext{
1 A escala de Likert utilizada no questionário ROSE varia de 1 a 4 pontos sendo, portanto, o valor médio ou neutro de 2,5.
} 


\subsection{Análise de dados}

Os resultados obtidos dos questionários ROSE foram analisados utilizando o software IBM SPSS, este é um programa estatístico bastante utilizado pelas áreas das ciências sociais e que nos possibilita realizar as análises estatísticas necessárias para o desenvolvimento do trabalho. Para responder o objetivo utilizaremos as análises de tabulação cruzada e qui-quadrado, descritas abaixo. Dos 2.365 respondentes do questionário, 2.235 responderam a ambas as questões e formam a amostra deste estudo.

A tabulação cruzada é uma análise descritiva dos dados, cuja principal finalidade é cruzar a frequência de respostas entre duas variáveis, esclarecendo o comportamento do grupo de estudo em relação a essas variáveis, permitindo criar agrupamentos. Utilizamos essa análise para cruzar as respostas dos jovens brasileiros para as questões F2 interesse absoluto, com a F5 interesse relativo. Buscando compreender, desta maneira, como as respostas para essas questões interagem.

Por sua vez, a análise de qui-quadrado foi utilizada para verificar se os grupos formados pela tabulação cruzada podem ser considerados grupos verdadeiros, ou seja, grupos que respondem de maneira distintas entre si. O qui-quadrado é um teste não paramétrico utilizado para mensurar dependência entre duas variáveis, avaliando se estas são ou não homogêneas (Hair, et al., 2009). Valores iguais ou maiores que 0,05 indicam que as variáveis são homogêneas $(\mathrm{H} 0)$, valores menores que 0,05 indicam que as variáveis são diferentes ou heterogêneas (H1). A hipótese $\mathrm{H} 0$ considera os agrupamentos como estatisticamente iguais, ou seja, respondem de maneira semelhante, não fazendo sentido separar o objeto estudado em grupos. Já a Hipótese H1 considera que os objetos em estudo são diferentes estatisticamente formando grupos distintos.

\section{Resultados e Discussão}

Quando combinamos, por meio da tabulação cruzada, as respostas dos jovens brasileiros para as questões " $A$ disciplina de ciência é interessante" (F2) e "Gosto mais de ciência que de outras disciplinas" (F5) encontramos o resultado que pode ser visualizado na Tabela 1.

Tabela 1 - Tabulação cruzada entre as respostas dos jovens brasileiros para a questão F2 e F5 do questionário ROSE.

\begin{tabular}{|c|c|c|c|c|c|c|}
\hline & & \multicolumn{4}{|c|}{ F5 "Gosto mais de ciência que de outras disciplinas" } & \multirow[b]{2}{*}{ Total } \\
\hline \multirow{7}{*}{$\begin{array}{c}F 2 \text { "A disciplina de } \\
\text { ciência é } \\
\text { interessante" }\end{array}$} & & $\begin{array}{l}\text { Discordo } \\
\text { totalmente }\end{array}$ & Discordo & Concordo & $\begin{array}{l}\text { Concordo } \\
\text { totalmente }\end{array}$ & \\
\hline & $\begin{array}{l}\text { Discordo } \\
\text { totalmente }\end{array}$ & 130 & 24 & 10 & 7 & 171 \\
\hline & Discordo & 194 & 105 & 59 & 13 & 371 \\
\hline & Concordo & 231 & 201 & 116 & 48 & 96 \\
\hline & Concordo & & & & & \\
\hline & totalmente & 346 & 231 & 236 & 284 & 1097 \\
\hline & Total & 901 & 561 & 421 & 352 & 2.235 \\
\hline
\end{tabular}

Fonte: Autores.

Para melhor compreender esses resultados agrupamos as respostas dos jovens brasileiros formando quatro grupos descritos na Figura 2. A nomenclatura utilizada para denominar os grupos é uma adaptação de Ogawa e Shimode (2004). 
À direita no eixo F2 e acima no eixo F5 encontram-se as respostas "Concordo Totalmente" e "Concordo" nas respectivas questões. Analogamente, abaixo no eixo F5 e à esquerda no eixo F2 estão agrupados os estudantes que assinaram "Discordo" e "Discordo Totalmente" das respectivas questões.

Figura 2 - Distribuição dos Grupos em relação ao interesse pela disciplina Ciência.

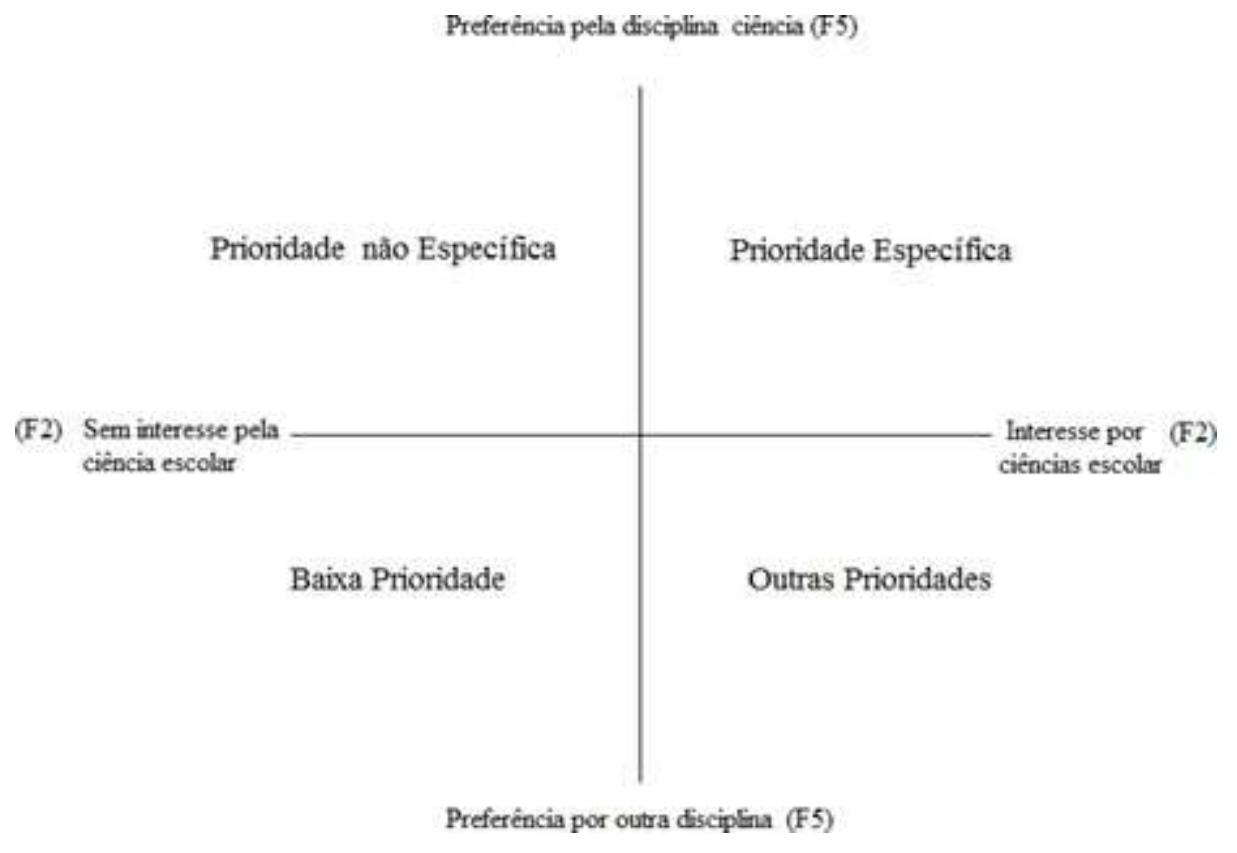

Fonte: Autores, adaptado de Ogawa e Shimode (2004)

Os grupos reúnem os seguintes perfis de jovens brasileiros:

- Grupo Prioridade Específica: Jovens que consideram a disciplina ciências interessante e que gostam mais de ciências do que de outras disciplinas. Esses respondem concordo ou concordo totalmente para ambas as perguntas. Podemos descrever estes jovens como aqueles que têm na disciplina de ciências sua preferência;

- Grupo Outras Prioridades: Jovens que consideram a disciplina interessante, porém gostam mais de outras disciplinas em detrimento da disciplina ciências. Responderam concordo ou concordo totalmente para F2 e discordo ou discordo totalmente para F5. Mesmo que estes jovens gostem da disciplina de ciências, esta não é sua disciplina favorita;

- Grupo Prioridades não Específica: Gostam mais de ciências que de outras disciplinas, porém não acham a disciplina interessante. Esses responderam concordo ou concordo totalmente para F5 e discordo ou discordo totalmente para F2. Nesse grupo encontramos um paradoxo entre as respostas, pois mesmo não achando a disciplina interessante, eles a preferem.

- Grupo Baixa Prioridade: Não consideram a disciplina ciência interessante e preferem outras disciplinas em detrimento da disciplina de ciências. Estes responderam discordo ou discordo totalmente para ambas as perguntas. Ao contrário do grupo Prioridade não específica aqui encontramos uma coerência entre as respostas, pois se o jovem não tem interesse em ciências é natural que esta não seja sua disciplina favorita. A distribuição desses grupos se encontra na Tabela 2. 
Tabela 2 - Distribuição dos jovens brasileiros nos grupos pelo interesse na disciplina ciências.

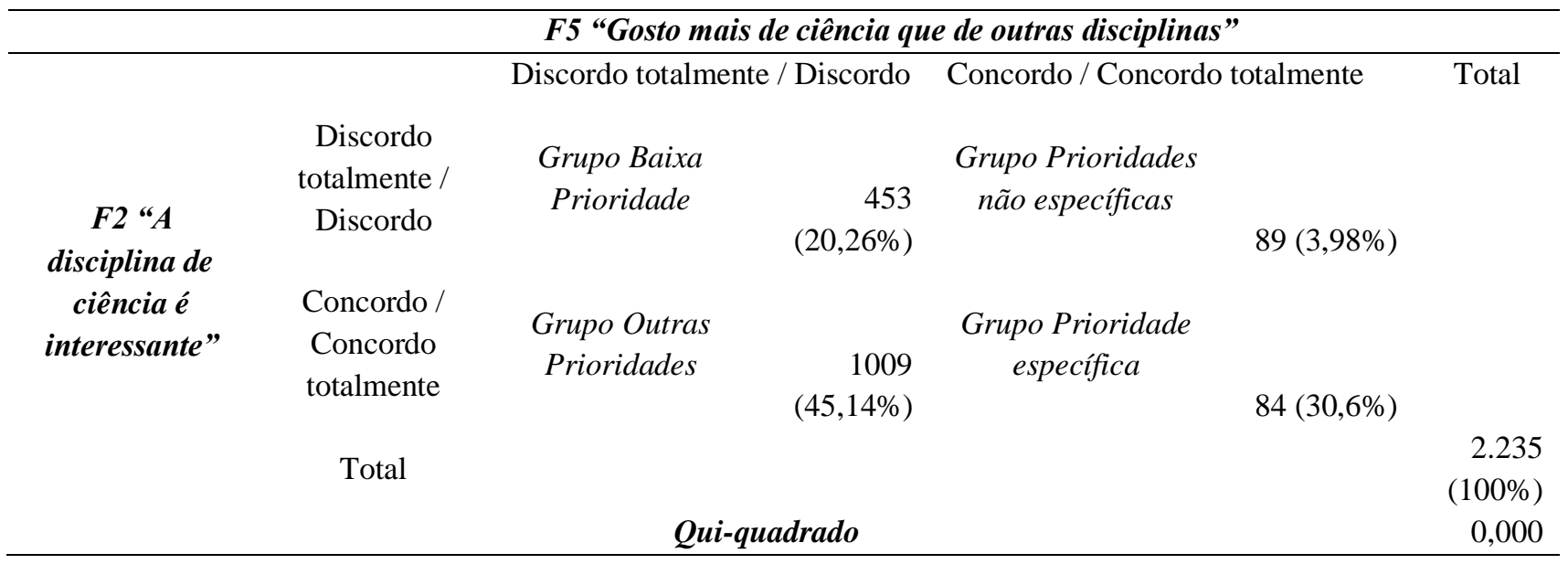

Fonte: Autores.

O grupo Prioridades não específicas, demonstrou ser o grupo menos representativo dentre os jovens brasileiros (3,98\%). Ogawa e Shimode (2004) acreditam que o grupo de prioridades não específica, o qual também foram os menos representativos dentre os jovens japoneses $(2,4 \%)$, gostam da metodologia das aulas de ciências, como ir ao laboratório e realizaram experimentos, mas não possuem interesse nos conteúdos abordados pela disciplina ciências (Ogawa \& Shimode, 2004).

Não podemos dizer que este caso se aplique aos alunos brasileiros, segundo Borges (2002) muitos jovens brasileiros nunca tiveram a oportunidade de entrar em um laboratório de ciências, seja por falta de laboratório ou por falta de recursos e manutenção nesses espaços em suas escolas. Mesmo que o uso de laboratórios não seja uma prática frequente no Brasil como afirmado pelo autor por Borges (2002), jovens que tiveram contado com práticas experimentais relatam que estas os ajudam na aprendizagem da disciplina (Farias, et al., 2020) Sabemos que os jovens brasileiros reconhecem a importância dos seus professores de ciências na mediação da aprendizagem (INEP, 2016) e que em sua maioria recorre a eles para conversar sobre assuntos da área da C\&T, vendo no professor uma fonte confiável (INCT-CPCT, 2019). Podendo, assim, o professor ser um ponto importante que explica o comportamento desse grupo.

Ao contrário do grupo anterior, os jovens de Prioridades específicas representam um montante significativo dos estudantes brasileiros $(30,6 \%)$. Mesmo possuindo realidades muito diferentes, o valor encontrado no contexto brasileiro se aproxima do mesmo resultado encontrado para os jovens japoneses (33,0\%) (Ogawa \& Shimode, 2004). Esses resultados nos mostram que praticamente todos os jovens brasileiros que possuem um alto interesse relativo pela disciplina ciências também a considera interessante. Porém dentre aqueles que têm um alto interesse absoluto pela disciplina a maioria demonstra ter um baixo interesse relativo pela disciplina, fazendo parte do grupo Outras prioridades.

Como vimos, o grupo Outras prioridades representa aproximadamente 45,14\% dos jovens brasileiros, porcentagem maior do que a alcançada pelos jovens japoneses de 31,7\% (Ogawa \& Shimode, 2004). Essa alta porcentagem está ligada com o alto interesse dos jovens brasileiros pela disciplina ciências e sua baixa preferência pela disciplina em detrimento de outras (Santos-Gouw, 2013; Santos-Gouw \& Bizzo, 2016). Schreiner e Sjøberg (2007) demonstraram que jovens de países em desenvolvimento tendem a considerar a disciplina ciências mais interessante, do que os jovens de países desenvolvidos. Nesse quesito, segundo Santos-Gouw (2013), o Brasil segue a tendência dos países em desenvolvimento. Garcia e Bizzo (2015) em pesquisa envolvendo estudantes brasileiros pertencentes ao $5^{\circ}$ ano do ensino fundamental, constatou que os alunos apontam português e matemática como as disciplinas mais relevantes, a posição da disciplina ciências varia entre terceiro e quinto lugar. 
Vale ressaltar alguns pontos importantes em que o estudo realizado por Garcia e Bizzo (2015) difere do proposto aqui, mas sem tornar sua comparação descabida: 1- Contexto distante da realidade brasileira - os estudantes contemplados pela pesquisa dos autores pertencem a uma das regiões brasileiras com maior IDH (Índice de Desenvolvimento Humano) e suas escolas possuem uma alta pontuação no IDEB (Índice de Desenvolvimento da Educação Básica); 2 - Os alunos elencaram a relevância e não preferência pelas disciplinas - apenas seis disciplinas foram consideradas para esse ranqueamento, português, matemática, ciências, história, geografia e inglês. Para os autores esse alto valor atribuído às disciplinas de português e matemática sofre influência dos professores e diretores, uma vez que o desempenho dos estudantes nessas disciplinas compõem diretamente o IDEB da escola.

O IDEB foi criado em 2007 e em seu cálculo leva em consideração o censo escolar - índice de aprovação, reprovação e evasão escolar - e a média de desempenho no SAEB (Sistema de Avaliação da Educação Básica). O SAEB é composto por uma avaliação em larga escala que mensura a qualidade da educação básica no Brasil. Surgiu em 1990, e a partir do ano 2000 passou a avaliar os estudantes em finais de ciclos escolares ( $5^{\circ}$ ano e $9^{\circ}$ ano do ensino fundamental e $3^{\circ}$ ano do ensino médio) nas disciplinas de português e matemática. Esse enfoque maior atribuído a português e matemática pelo SAEB e pelo IDEB e que recai sobre os professores e diretores das escolas pode influenciar na percepção dos alunos acerca das disciplinas.

Jenkins e Nelson (2005) ressaltam que precisamos compreender que, apesar dessa dicotomia entre os interesses pela disciplina ciências que são encontrados em diferentes países, os contextos socioeconômicos, culturais e do próprio currículo de ciências das diferentes nações devem ser levados em conta. Os jovens brasileiros do grupo Outras prioridades ilustram bem essa dicotomia. Essa rejeição que a disciplina ciências sofre quando comparada a outras pode estar ligada ao método de ensino ou ao conteúdo contemplado pela disciplina. Segundo Pozo e Crespo (2009) esses são os motivos para a crise que a educação em ciência está vivendo, aulas e currículo que pouco mudaram ao longo do tempo indo de encontro a uma sociedade com uma demanda formativa muito diferente daquela para a qual esse currículo foi feito.

O livro didático permeia (e confunde-se com) o currículo, uma vez que determina o conteúdo e o ritmo do que deve ser abordado, media o processo de ensino-aprendizagem em sala de aula, assim como muitas vezes é o único suporte didático para alunos e professores (Amestoy \& Tolentino-Neto, 2017). Esses autores verificaram que apesar de os livros abordarem a maioria dos interesses de aprendizagem em ciências dos jovens brasileiros, o método da abordagem às vezes é tradicional/propedêutico demais e se distancia muito do que é previsto pelas Diretrizes Curriculares Nacionais.

Por fim, o grupo Baixa prioridade representa 20,3\% dos jovens brasileiros, número inferior ao encontrado nos jovens japoneses (33\%) por Ogawa e Shimode (2004). Esse grupo não demonstra nem interesse relativo, nem interesse absoluto na disciplina ciência, podemos dizer que levando em conta apenas esses parâmetros jovens desse grupo encontram-se mais próximos aos jovens de países desenvolvidos.

Os jovens japoneses pertencentes a esse grupo são os que menos realizam atividades relacionadas à ciência e tecnologia fora da sala de aula, são também, os que menos demonstram ter interesse em aprender tópicos relacionados à disciplina de ciências (Ogawa \& Shimode, 2004). Porém os autores encontraram alguns tópicos em que esse grupo demonstra mais interesse (uso e funcionamento de celulares) os quais podem servir como tema motivador para tornar as aulas de ciências mais atrativas para esses alunos (Ogawa \& Shimode, 2004.)

Nesse artigo procuramos compreender como os jovens brasileiros se dividem frente a sua preferência pela disciplina ciências, para isso cruzamos duas questões: "A disciplina ciências é interessante" e "Gosto mais de ciências que de outras disciplinas", obtendo assim os quatro grupos descritos nesse trabalho. Os interesses em aprendizagem parecem ser engrenagens capazes de motivar os alunos (Ogawa \& Shimode, 2004; Ocampo \& Tolentino-Neto, 2020), sendo assim, acredita-se que investigar os interesses na aprendizagem dentro de cada grupo encontrado nesse estudo pode ser de grande valia para motivar os alunos dos grupos com baixa preferência pela disciplina ciências em suas aulas. 


\section{Considerações Finais}

Os jovens brasileiros apresentam um alto interesse absoluto e um baixo interesse relativo pela disciplina ciências. Por um lado, esses jovens vão na contramão de seus pares de países desenvolvidos. Por outro lado, concordam ao não atribuírem à disciplina ciências uma predileção em relação às demais disciplinas. Essa baixa preferência pela ciência escolar pode ter relação com a diminuição pela busca ou desejo de seguir carreiras científicas (Vázquez \& Manassero, 2008). Não se pode dizer, sem maiores investigações, o que foi causa e o que é efeito.

Quando tentamos compreender o comportamento dos jovens brasileiros em relação a sua preferência pela disciplina ciências observamos a formação de quatro grupos: Prioridades não específicas, Prioridades específicas, Outras prioridades e Baixa prioridade. Sendo os três últimos os mais expressivos na amostra brasileira.

O grupo Baixa prioridade não prefere a disciplina ciências em nenhum nível, tendo um baixo interesse absoluto e relativo. Já o grupo Prioridades específicas tem uma alta preferência pela ciência escolar, o que pode indicar que este grupo possua uma tendência maior para seguir carreira ou estudos na área da C\&T. Para corroborar com essa hipótese seriam interessantes estudos futuros sobre esse tema.

O grupo Outras prioridades chama atenção, primeiramente pela sua grande expressividade dentre os jovens brasileiros, mas também por nos fazer questionar o que difere os grupos Outras prioridades e Prioridades específicas? Esses jovens possuem os mesmos interesses na aprendizagem de ciências? Como percebem as suas aulas de ciência? As experiências fora da escola com a temática C\&T são a mesma?

Seria interessante, em trabalhos futuros, investigar dentre os jovens brasileiros pertencentes a esse grupo possíveis temas motivadores, os quais podem vir a ser utilizados nas aulas de ciências para tornar as aulas mais atrativas para esse grupo. As respostas a essas questões podem nos levar a compreender melhor o comportamento dos jovens de cada grupo, servindo de subsídio para os professores buscarem pontos de convergência entre esses grupos tornando a disciplina ciências mais atrativa para os jovens brasileiros. É nas políticas públicas e nas ações baseadas em evidências como estas, que nascem propostas que levem a um movimento, gradual e sustentável, de jovens aos grupos mais simpáticos às temáticas da ciência. Professores e recursos didáticos que colaboram na formação (inicial e continuada) de professores encontram aqui um elemento dissertativo importante no avanço da valorização do ensino tecnológico-científico.

\section{Referências}

Amestoy, M., B., \& Tolentino-Neto, L., C., B. (2017). Articulações entre o livro didático de biologia e os interesses dos estudantes do ensino médio. Revista Dynami, FURB, 23(2), 90-195.

Borges, A., T., (2002). Novos Rumos para o laboratório escolar de ciências. Caderno Brasileiro de Ensino em Física, 19(3), 291-313.

Freitas, M., B, de, Parente, S., R., Bastos, R., S., Silva, W., F. de, Ferreira, F., M., Alencar, D., B., de, Lima, B., A., V. \& Barros, I., N. (2020). A importância do uso de experimentos científicos para o ensino de Ciências no Ensino Fundamental: um estudo de caso. Research, Societu and Development, 9(7), 1-15, e311974159. http://dx.doi.org/10.33448/rsd-v9i7.4159.

Garcia, P., S., \& Bizzo, N. (2015). Educational policies and Science education in Brazil: a case study. In: European Science Education Research Association (ESERA), Helsinki.

Gatti, B., A. (2004). Estudos quantitativos em educação. Educação e Pesquisa, 30(1), 11-30.

Gil, A., C. (2002). Como elaborar projetos de pesquisa, (4a ed), Atlas S. A.

Hair, J., F., Black, W. C., Badin, B., J., Anderson, R., E. \& Tatham, R., L. (2009). Análise Multivariada de Dados. Tradução de Adonai Schlup Sant'Ana. 6. Ed.: Bookman.

Instituto Nacional de Ciência e Tecnologia em Comunicação Pública da Ciência e Tecnologia (INCT-CPCT) (2019). O que os jovens brasileiros pensam da ciência e da tecnologia? Resumo executivo. http://www.coc.fiocruz.br/images/PDF/Resumo\%20executivo\%20survey\%20jovensFINAL.pdf.

Instituto Nacional de Estudos e Pesquisas Educacionais Anísio Teixeira (INEP) (2008). Brasil no PISA: Sumário Executivo. http://portal.inep.gov.br/pisa-nobrasil. 
Research, Society and Development, v. 10, n. 5, e8210514549, 2021

(CC BY 4.0) | ISSN 2525-3409 | DOI: http://dx.doi.org/10.33448/rsd-v10i5.14549

Instituto Nacional de Estudos e Pesquisas Educacionais Anísio Teixeira (INEP) (2016). Brasil no PISA: Sumário Executivo. http://download.inep.gov.br/acoes_internacionais/pisa/documentos/2016/pisa_brasil_2015_sumario_executivo.pdf.

Jenkins, E., W., \& Nelson, N., W. (2005). Important but not for me: students' attitudes towards secondary school science in England. Research in Science \& Technological Education, 23(1), 41-57.

Kearney, C. (2016). Efforts to increase students' interest in pursuing mathematics, science and technology studies and careers. National measures taken by 30 countries - 2015 report. Brussels: European Schoolnet.

Kearney, C. (2011). Efforts to Increase Students' Interest in Pursuing Science, Technology, Engineering and Mathematics Studies and Careers, European. National Measures taken by 21 of European Schoolnet's Member Countries - 2011 Report. European Schoolnet.

Matthewa, P. (2007). The Relevance of Science Education in Ireland. Dublin: Royal Irish Academy. http://www.ria.ie/publications/rose.html.

Minayo, M., C. (2000). Pesquisa Social: Teoria, Método e Criatividade, (17a ed), Vozes.

Ocampo, D., M., \& Tolentino-Neto, L., C., B., de. (2019). Cluster Analysis for Data Processing in Educational Research. Acta Scientiae (ULBRA), $21,34-48$. doi: $10.17648 /$ acta.scientiae.v21iss4id5119.

Ocampo, D., M., \& Tolentino-Neto, L., C., B., de. (2020). As diferentes tipologias que descrevem o interesse dos jovens brasileiros pelas ciências. Amazônia: Revista de Educação em Ciências e Matemáticas, 16(37), 164-176. https://www.periodicos.ufpa.br/index.php/revistaamazonia/article/view/8660/6696.

Ogawa, M. \& Shimode, S. Three distinctive groups among japanese students in terms of their school science preference: from preliminary analysis of japanese data of an international survey 'the relevance of science education' (ROSE). (2004). Journal of Science Education in Japan, 28(4), $279-291$.

Pozo, J., I. \& Crespo, M., A., G. (2009). A aprendizagem e o ensino de ciências: do conhecimento cotidiano ao conhecimento científico. (5a ed), Artmed.

Santos-Gouw, A., M. (2013). As opiniões, interesses e atitudes dos jovens brasileiros frente à ciência: uma avaliação em âmbito nacional. Tese em (Ensino de Ciências e Matemática) Faculdade de Educação, Universidade de São Paulo, São Paulo.

Santos-Gouw, A., M. \& Bizzo, N. (2016). A percepção dos Jovens brasileiros sobre suas aulas de ciências. Educar em Revista, 60, 277-292.

Schreiner, C. (2006). Exploring a ROSE-garden: Norwegian youth's orientations towards science - seen as signs of late modern identities. Doctoral thesis, in (Teacher Education and School Development) Faculty of Education, University of Oslo, Oslo.

Schreiner, C. \& Sjøberg, S. (2004) Sowing the Seeds of Rose: Background, rationale, questionnaire development and data collection for ROSE (The Relevance of Science Education) - a comparative study of students' views of science and science education. Acta Didactica. University of Oslo, Oslo. https://roseproject.no/key-documents/key-docs/ad0404-sowing-rose.pdf.

Sjøberg, S. (2004). Science Education: The voice of the learners. Brussels. http://roseproject.no/network/countries/norway/eng/nor-sjoberg-eu2004.pdf.

Stefánsson, K., K. (2006) 'I just don't think it's me' A study on the willingness of Icelandic learners to engage in Science related issues. Master's thesis, in (Science Education in the Department of Teacher Education and School Developmente). University of Oslo, Oslo.

Tolentino-Neto, L., C., B., de. (2008) Os interesses e posturas dos alunos frente às ciências: resultados do Projeto ROSE aplicado no Brasil. Tese em (Ensino de Ciências e Matemática) Faculdade de Educação, Universidade de São Paulo, São Paulo.

Vázquez, A., A. \& Manassero, M., A., M. (2008). El declive de las actitudes hacia la ciência de los estudiantes: um indicador inquietante la educación científica. Revista Eureka sobre Enseñanza y Divulgación de las Ciencias, 5(3), 274-292. 